\title{
Téoros
}

Revue de recherche en tourisme

\section{Storyville et le tourisme sexuel}

Une tradition inventée?

\section{Martine Geronimi}

Volume 22, numéro 1, printemps 2003

Tourisme et sexualité

URI : https://id.erudit.org/iderudit/1071583ar

DOI : https://doi.org/10.7202/1071583ar

Aller au sommaire du numéro

Éditeur(s)

Université du Québec à Montréal

ISSN

0712-8657 (imprimé)

1923-2705 (numérique)

Découvrir la revue

Citer cet article

Geronimi, M. (2003). Storyville et le tourisme sexuel : une tradition inventée ? Téoros, 22(1), 36-43. https://doi.org/10.7202/1071583ar

Ce document est protégé par la loi sur le droit d'auteur. L'utilisation des services d'Érudit (y compris la reproduction) est assujettie à sa politique d'utilisation que vous pouvez consulter en ligne.

https://apropos.erudit.org/fr/usagers/politique-dutilisation/
Cet article est diffusé et préservé par Érudit.

Érudit est un consortium interuniversitaire sans but lucratif composé de l’Université de Montréal, l'Université Laval et l'Université du Québec à Montréal. Il a pour mission la promotion et la valorisation de la recherche. https://www.erudit.org/fr/ 


\section{Storyville et le tourisme sexuel Une tradition inventée?}

\section{Martine Geronimi}

a réputation de la Nouvelle-Orléans re$\checkmark$ pose sur son patrimoine français, mais également dans l'esprit des touristes américains et internationaux, qui la fréquentent depuis près de deux siècles, sur la légèreté de ses mœurs françaises et catholiques. Le carnaval, qui atteint son paroxysme à Mardi Gras, capitalise encore l'imaginaire de permissivité absolue pour les visiteurs en mal d'Ailleurs et d'exotisme sexuel. C'est durant la période de la prostitution légalisée à la fin du XIX ${ }^{\mathrm{e}}$ siècle que s'est forgée cette notoriété licencieuse. Le quartier de Storyville était le haut lieu du tourisme sexuel. Les maisons de tolérance, vantées par les guides touristiques et les annonces classées dans les journaux, ont aujourd'hui disparu. Mais le nom de Storyville reste associé à ce parfum de débauche auréolée de la naissance du Jazz.

L'image de Storyville est encore présente dans la mémoire des voyageurs contemporains. Ce nom renvoie au lieu de naissance du Jazz, dans cette Nouvelle-Orléans permissive du début du $\mathrm{XX}^{\mathrm{e}}$ siècle ${ }^{1}$. Il est toutefois remarquable que peu de vestiges ne subsistent dans le paysage urbain néo-orléanais. Les archives, comme les bâtisses, ont été éradiquées des traces qui auraient pu alimenter la mémoire historique de la ville. La plupart des souvenirs proviennent de la mémoire collective (Rose, 1974) et de quelques documents conservés au Musée Mint et au William Research Center ${ }^{2}$.

Je soutiens dans cet article que les liens entre un lieu mythique, reconnu pour son libertinage français et catholique, et un domaine festif portuaire en pleine expansion économique $^{3}$, ont conduit à la naissance d'un quartier de prostitution légalisée à l'échelle du continent nord-américain : Storyville à la Nouvelle-Orléans entre 1898 et 1917.

Cet espace, fantasmé par les visiteurs masculins, a donné naissance à une littérature de voyage, sous la forme de guides et de journaux, nouvelles figures d'annonces publicitaires, qui vantaient la destination pour son attraction principale : le commerce de la sexualité libéralisée, ce que nous nommons, de nos jours, le tourisme sexuel.

Ma vision du tourisme sexuel dans cette fin $\mathrm{du} \mathrm{XIX}^{\mathrm{e}}$ siècle, repose sur une définition précise du phénomène. Il s'agit de voyageurs séjournant à la Nouvelle-Orléans qui recherchaient expressément des relations vénales avec des jeunes filles ou jeunes femmes, retenues dans des maisons dites de tolérance. L'espace de la NouvelleOrléans ne semble pas anodin, si on le met en parallèle avec les pratiques de libertinage attribuées aux Français et aux Créoles. Il convient également de se souvenir que ce port était un marché d'esclaves réputé jusqu'à la fin de la guerre de Sécession ${ }^{4}$.

La pratique de la prostitution est attestée dans tous les ports de la planète depuis fort longtemps (Vanaoyeke, 1990 ; McGinn, 1998). Une ville portuaire comme la Nouvelle-Orléans abondait en femmes exerçant ce métier bien avant la création de Storyville ${ }^{5}$. Scandalisés par la diffusion des quartiers aux lanternes rouges un peu partout dans la ville, les élites ont voulu préserver la distinction du centre historique, le French Quarter ou Vieux Carré et leur environnement bourgeois. Ils ont ainsi créé sur le sol américain, un lieu hors temps et horsloi, une enclave légale dans laquelle, des jeunes femmes voire de très jeunes filles étaient autorisées à se prostituer aux touristes américains et internationaux de passage. Cette prostitution « encadrée » se déroulait dans des maisons closes sises dans un quartier à la frontière du French Quarter.

Comme la Nouvelle-orléans est réputée pour son Carnaval, il est important de montrer les liens existants entre cette attraction majeure, pendant laquelle tous les comportements d'inversion sociale et de transgression sont tolérés, et la fréquentation de la ville par des touristes recherchant une expérience hors du temps de leur vie ordinaire, dans un lieu lointain et mystérieux, espace d'évasion et de totale licence.

Cet article présente dans un premier temps l'espace géographique de Storyville tel qu'il existait de 1898 à 1917. Dans un second temps, c'est l'activité du quartier qui est montrée en mettant l'accent sur les acteurs principaux. Enfin, le moment du carnaval est analysé au travers de sa publicité spécialisée et de ses codes.

Le but de cet article est d'une part de remettre en mémoire, de la manière la plus scientifique possible, un moment du passé en lien avec notre présent. D'autre part, cet article veut faire réfléchir sur les espaces de marginalité sociale ou géographique qui touchent à l'imaginaire de l'exotisme charnel. Il s'agit de montrer qu'une industrialisation d'une forme de services aux voyageurs s'est mise en place dès la fin du $\mathrm{XIX}^{\mathrm{e}}$ siècle, profitant des quartiers de prostitution légalisée, créant ainsi une « nouvelle tradition $»^{6}$ : le tourisme sexuel. 


\section{Un espace géographique bien défini, le quartier de Storyville et son Red-Light}

Le nom du quartier tire son origine du patronyme d'un certain Alderman Sydney Story, conseiller de la Nouvelle-orléans et respectable homme d'affaires préoccupé par le problème de la prostitution dans cette fin du XIX ${ }^{\text {ème }}$ siècle. Soutenu par l'Attorney Thomas McCaleb Hyman, il entreprit une enquête sur la possibilité de réduire les nuisances de « ce mal nécessaire » qu'était devenue la prostitution urbaine. Il avait sillonné l'Europe en vue d'une solution qui limiterait l'extension de la prostitution à l'intégralité de la ville. L'Attorney avait, quant à lui, fait toutes les recherches légales. De retour, Story proposait, au début de 1897, une ordonnance qui limiterait la prostitution à un quartier spécifique, installé à la limite nord du French Quarter ou Vieux Carré (figure 1).

Cette législation répondait à une demande des résidents de la Nouvelle-Orléans qui, depuis longtemps, se plaignaient des nuisances causées par le grand nombre de prostituées. Ces plaintes de citoyens respectables étaient notamment alimentées par

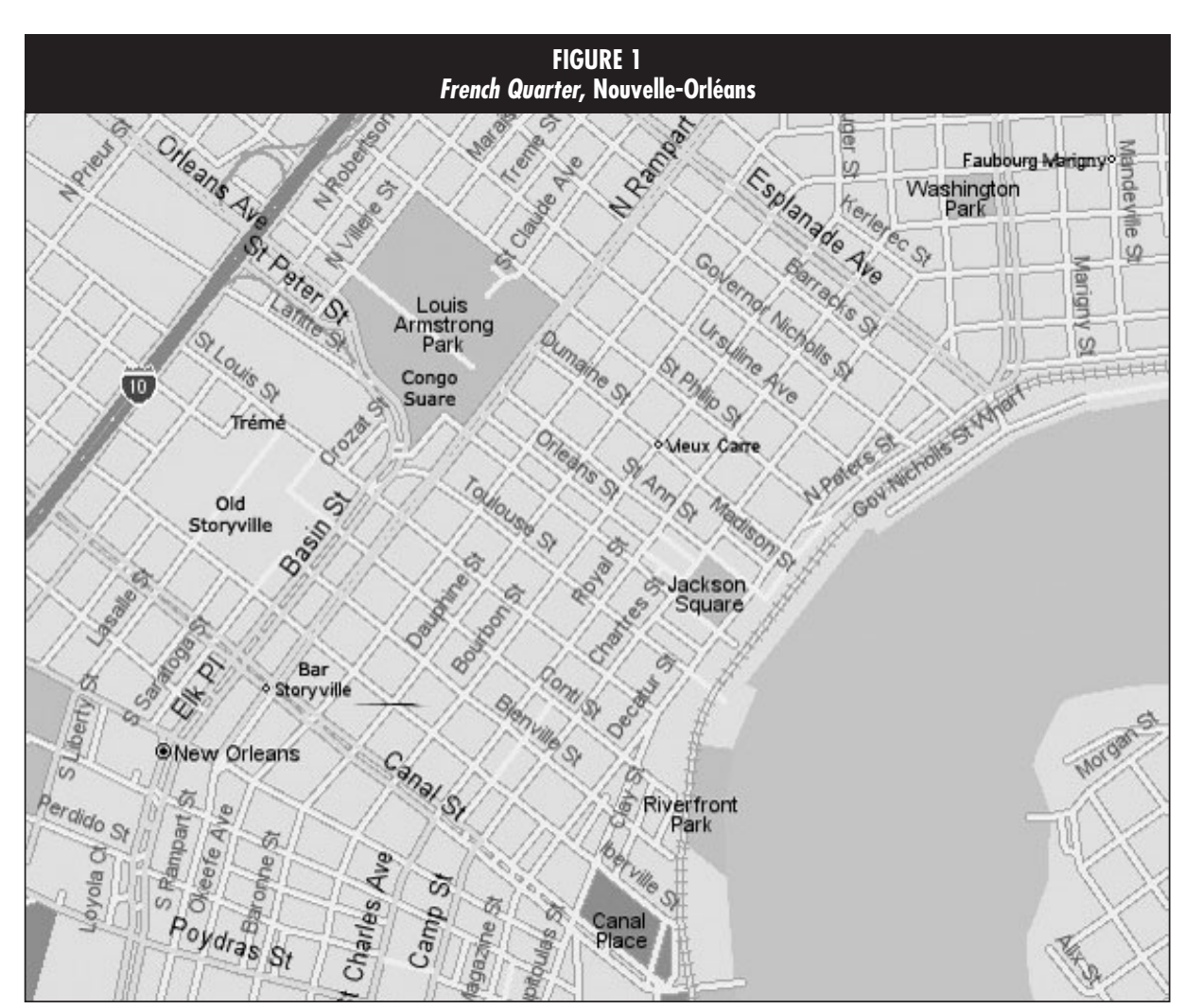

une revue hebdomadaire intitulée The Mascot, qui à partir de 1882 , et, régulièrement durant une dizaine d'années, dénonçait le vice et la prostitution dans la ville. Derrière ce journal à scandales se profilait un mouvement moralisateur qui allait jusqu'à proposer, bien avant la réalisation légale de Storyville, un règlement comparable. Le 26 mars 1887, les citoyens de la Nouvelle-Orléans pouvaient lire dans les colonnes du journal que « le remède approprié était de définir les contours d'un quartier délimitant une zone de prostitution en dehors de laquelle il serait illégal d'exercer à titre de prostituée » $($ Rose 1974,39) 7 .

C'est au Nouvel An 1898 que fut institutionnalisée cette première expérience d'un quartier officiel de la prostitution. Ce fut la presse qui, par dérision, baptisa ce quartier du nom du conseiller. Et, bien malgré lui, son nom reste dans la mémoire collective attaché à la réputation d'un quartier pour le moins festif.

Le quartier de Storyville doit se comprendre dans le cadre social et culturel de l'époque. Les années 1870 ouvrent l'Ėre victorienne avec sa morale stricte et son hypocrisie concernant la sexualité. Il faut éviter de contaminer les femmes respectables par la vue des courtisanes et autres «femmes perdues ». En même temps, il n'est pas question d'éliminer la prostitution qui est jugée comme étant un mal nécessaire. C'est pourquoi les législateurs décident de contenir la prostitution dans des limites précises et de créer des ghettos éclairés les nuits appelés Red Light districts dans de nombreuses villes américaines. Ainsi, San Francisco comptait 83 maisons de tolérance en 1917 , lors de leur fermeture, de même Galveston comptait encore en 1929 plus de 50 maisons de tolérance illégales en activité.

Cette période d'industrialisation et d'urbanisation intenses aux changements sociaux profonds se caractérisait par une fort intérêt pour l'hygiène et la santé. Aussi la nécessité de réglementer la prostitution prenait sa source dans la nécessité de la prévention des maladies sexuellement transmissibles, comme la syphilis qui causait de graves ravages. Ce mouvement de réformes menées par des autorités médicales et des chercheurs utilisaient les statistiques, nouvelles sciences, qui étayaient leurs convictions.

À côté de ce mouvement réformateur hygiéniste, existait aussi un mouvement d'éradication de la prostitution. Celui-ci, appelé « social purity », cherchait à « abolir le vice ». Ces réformateurs menés par des principes moraux utilisèrent également les statistiques pour justifier leurs points de vue. Malgré des divergences entre les réformateurs, hommes ou femmes, la tendance globale voulait qu'il s'intéressât à la prostitution urbaine des Red Lights. La grande visibilité de ces lieux perturbait et donna naissance à des organisations antiprostitution dans toute l'Amérique (Rosen, 1982 ; Conelly, 1980).

Ce n'est qu'à partir des années 1910 que les groupes anti-prostitution commencent a gagner leur bataille de suppression des Red Lights. En 1917, la croisade anti-prostitution trouve son meilleur défenseur en la personne de Newton D. Baker, Secrétaire d'état à la Guerre, qui interdit les quartiers de tolérance à proximité des bases militaires où s'entrâ̂naient les soldats en partance pour la Première guerre mondiale. Storyville est fermé par ordre du Secrétaire à la marine 
Josephus Daniels puisque le district se situait à moins de 5 miles des installations de l'Armée sises sur le Lac Pontchartrain, à Lakefront. Ainsi la santé des soldats devient le motif officiel à la fermeture d'un lieu de tolérance honni par la société puritaine.

\section{Une visite guidée dans Storyville aux alentours de 1914}

Les géographes s'intéressent aux plans des villes et, comme ce quartier n'existe plus, il est d'autant plus important de valider son existence par les traces laissées dans les textes et la mémoire collective. Notre plus grande source provient des travaux de Al Rose et de notre recherche, lors de la réalisation de notre thèse, tant aux archives de Loyola que celle d'UNO, du Musée Mint ou du William Research Center (Rose, 1974 ; Geronimi, 2001a).

Al Rose, dans son livre, nous propose un tour guidé de Storyville en 1914 (Rose 1974 : 73-96). Nous allons vous faire voyager dans cette Nouvelle-Orléans du début du $\mathrm{XX}^{\mathrm{e}}$ siècle et $\mathrm{y}$ intégrer Storyville telle qu'elle apparaissait aux visiteurs du carnaval de 1914. Cette année là est marquée par le premier défilé noir de Mardi Gras à l'initiative de la création du Zulu social Club (Geronimi, 2001b). Le terme de Jazz ne désigne encore pas cette musique devenue célèbre peu après; toutefois, Storyville est fréquenté par une myriade de musiciens noirs qui vont rester dans la mémoire collective. Si Storyville jouxte le Vieux Carré, il faut savoir que celui-ci ne cessait de se détériorer, qu'il ne bénéficiait plus de sa population originelle et était devenu le domaine des pauvres immigrés siciliens (Tregle, 1992). Il devint même un lieu de ruines qui exaltait le sentiment de nostalgie du passé perdu (Geronimi, 2001c).

Le Vieux Carré suivait le plan originel français et se délimitait selon trois axes principaux fermés par le Mississipi : à l'ouest la Rue Rampart, au nord l'avenue de l'Esplanade et au sud la rue Canal. Cette dernière œuvrait comme frontière entre la ville dite créole ou French Quarter et la ville américaine repérable par son CBD. Au nord de ce Quartier Français se trouvait une place célèbre Congo Square, lieu de danses réputé des esclaves du temps des créoles, avant la guerre de Sécession. Storyville était relégué au nord-ouest du Vieux Carré dans un quadrilatère largement délimité au nord par l'Avenue Clairborne et au sud par la rue Rampart. La rue Canal était une des limites et l'autre le Bayou St-John.

En général le visiteur arrivait par le train à la gare de dépôt Sud du côté de la ville américaine. Il pouvait, dès son arrivée, apercevoir les manoirs abritant les maisons de tolérance aux fenêtres desquelles se tenaient des prostituées. En effet, la ligne de chemin de fer longeait la rue Basin, l'artère principale de ce quartier et formait une coupure visible dans le paysage. Cette rue délimitait la zone sud du quartier et donc jouxtait le Vieux Carré, séparé de celui-ci par la rue Rampart, limite actuelle de ce quartier touristique. Plus spécifiquement, la limite nord de ce quartier réservé était la rue Robertson alors que la limite Est était la rue Saint-Louis et celle de l'Ouest était la rue Iberville (anciennement Customhouse).

En 1914, les maisons de prostitution étaient moins nombreuses qu'au temps de la création du quartier mais les Saloons, les cabarets et les dancings officiaient également en alternant les points d'intérêt pour les visiteurs en mal de dépaysement et d'expériences en tous genres. Quittant la gare et traversant l'artère commerçante principale, la rue Canal, le visiteur pouvait se rendre directement à pied sur Basin, afin d'y boire une bière dans un des saloons rencontrés sur son chemin comme le Milton J. Kelly's Terminal Saloon puis se rendre dans un cabaret pour écouter de la musique de la NouvelleOrléans. Il pouvait entendre le fameux refrain à la mode « Pretty Baby » et assister à une improvisation sur un air de Ragtime. Puis il se lançait à la recherche de plaisirs vénaux soit dans les maisons de tolérance de renom de la rue Basin, soit dans les différents garnis de la rue Iberville.

Aujourd'hui, à la place des trente huit blocs initiaux, il ne reste que cinq édifices de ce quartier détruit à la fin les années 30 pour laisser place à des habitations à loyers modérés du Iberville Housing Projects. Il semblerait qu'une grande nostalgie de cette époque romantisée hante les touristes contemporains qui se rabattent depuis les années 30 sur la rue Bourbon avec ses bars, ses cabarets et ses boîtes de Strip Tease (Vesey et Dimanche, 2003). Il nous faut souligner que ce quartier de la prostitution avait été repoussé dans le quartier de Trémé qui était un quartier peuplé par des résidents noirs. C'est ce même quartier qui ensuite sera défiguré par le passage de l'autoroute Interstate 10 afin d'épargner le Vieux Carré dans les années 70 (Lewis, 1976). Il abrite actuellement le Parc Louis Armstrong, sur l'emplacement de Congo Square.

\section{Déni de mémoire et réalité ; la prostituée et sa condition à la fin du XIXème siècle}

Selon les dires de Al Rose, en 1914, Storyville comptait pas moins de 750 femmes prostituées, 300 souteneurs, 200 musiciens, 500 domestiques et 150 employés de Saloon. Il nous affirme également qu'en 1898, il y avait en moyenne 2000 femmes engagées dans cette profession (Rose, 31 et 96).

Le grand nombre de prostituées à la Nouvelle-Orléans n'était pas une exception. Il correspond à un symptôme assez généralisé au XIX ${ }^{\mathrm{e}}$ siècle en Amérique comme en Angleterre. Ainsi dans ce dernier pays, dès 1841, il y aurait eu dans le Grand Londres de deux millions d'habitants, 55000 prostituées soit une prostituée pour 12 mâles adultes (Walkowitz, 1980).

La condition de la femme était très difficile dans une époque d'urbanisation galopante à un moment de changement sociétal induit par l'industrialisation et ses conditions salariales miséreuses (Moynihan, 1993). De nombreux sociologues se sont penché sur cette question. Il en ressort que la notion de femme déchue (Fallen women) est très importante (Winnifrith, 1994) et laisse à penser que plus d'une fois, les femmes aient eu à choisir entre « une vie de honte ou une vie de misère », comme le dénonçait la Déclaration des droits des femmes en 1876 à Philadelphie ${ }^{8}$.

Le recrutement à la Nouvelle-Orléans était grandement facilité par le fait que cette ville était une porte d'entrée pour les immigrants en provenance du monde entier (Rosen, 1990). Il nous faut désormais rappeler ce que la 
mémoire collective oblitère. Nous devons prendre conscience que cette prostitution, offerte aux touristes venant à la NouvelleOrléans, supposait un trafic de femmes, forme moderne non déguisée de l'esclavage. Or, même si cet aspect est documenté scientifiquement pour la période qui nous concerne, le grand public l'ignore et le touriste de ce début du $\mathrm{XIX}^{\mathrm{e}}$ siècle continue à peindre de couleurs romantiques et nostalgiques ce temps de libéralisation de la sexualité masculine.

Le trafic de femmes existe comme il a existé par le passé. Storyville est un des lieux particuliers en Amérique du Nord qui a accueilli ce type de trafic, mais il ne fut pas le seul puisque New York, Chicago, Montréal, Boston, Seattle ou San Francisco s'y sont illustré. Le trafic des femmes à travers l'Europe a été officiellement reconnu en 1877 lors d'une conférence internationale à Genève. En 1902, la France sous la pression de la réalité de la traite des Blanches, organisa une conférence internationale qui aboutit à un « arrangement international en vue d'assurer une protection efficace contre le trafic criminel connu sous le nom de traite des blanches » ${ }^{9}$. Cet agrément fut officiellement ratifié par le Président Roosevelt en 1908 et suivi d'une loi interdisant le trafic de personnes à travers les états ou en provenance de l'étranger, le Mann Act de 1910.

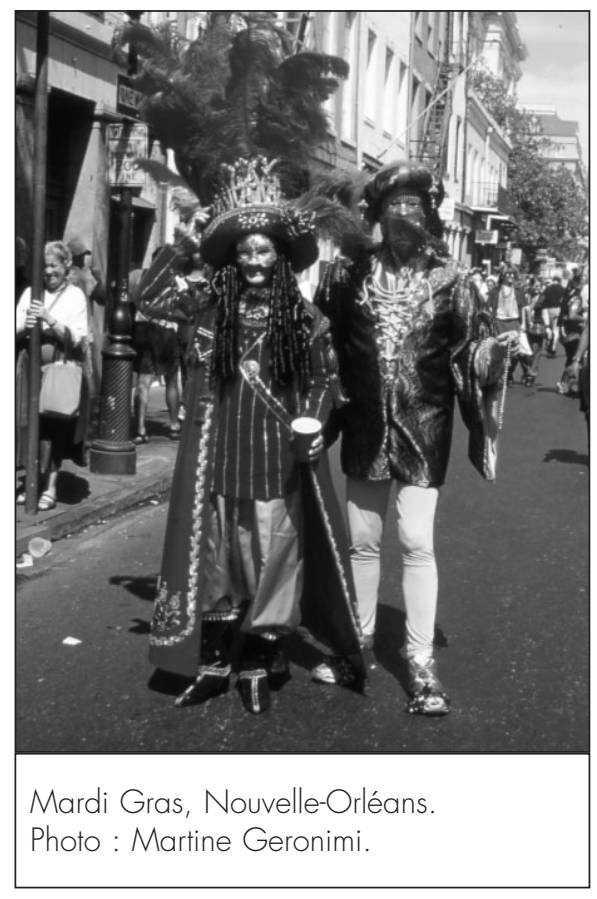

L'historienne Ruth Rosen nous révèle les chiffres suivants : de juin 1910 à janvier 1915, 1057 personnes furent condamnées aux États Unis pour trafic d'esclaves blanches. Elle nous précise que la Commission d'immigration au Sénat évalue à plusieurs milliers de femmes importées chaque année entre 1910 et 1915. Les détectives fédéraux précisaient que la moitié des femmes débarquaient à New York. Les trafiquants arrêtés étaient à 72,5\% des natifs Américains contre $11,5 \%$ des Italiens et 3,5\% des Russes. La grande majorité des femmes ou jeunes filles était recrutée soit dans le pays d'origine et entrait dans le pays comme épouse ou parente de l'homme qui l'accompagnait, ou bien ces jeunes femmes étaient repérées parmi les immigrantes qui arrivaient sur le Port de débarquement en Amérique, séduites puis vendues aux Madames, tenancières de maison close.

Il faut également admettre que la traite des femmes n'était pas uniquement le fruit de l'Internationalisation des échanges mais aussi celui de l'exode rural qui aboutissait à la prolétarisation des femmes dans les villes. De même, les changements sociaux semblent indiquer de nombreuses fugues chez les jeunes filles, notamment à New York. Il faut noter que l'esclavage pur avec extrême coercition représenterait un peu moins de dix pour cent des prostituées.

L'origine des prostituées de la NouvelleOrléans en 1900 était principalement Créole ou Noire. Créole signifiait, à l'époque des enquêtes, personne descendant des premiers colons d'origine européenne ou Canadienne-française. Les moins représentées dans la prostitution d'origine étrangère étaient les femmes juives, irlandaises et italiennes. Les Françaises étaient recherchées par toutes les maisons closes haut de gamme, tant à la Nouvelle-Orléans que dans tous les États-Unis.

\section{La vie à Storyville : Prospérité et entreprenariat}

Le film de Louis Malle, La petite (Pretty Baby), décrit en 1978 une facette de la prostitution à Storyville. Ainsi, Violet, douze ans, vit avec sa mère Hattie, une prostituée, et son petit frère Will, dans une de ces maisons de tolérance de Storyville, le quartier réservé de la Nouvelle-Orléans. On est en 1917, les États-Unis s'apprêtent à entrer dans la guerre et la marine américaine menace de fermer Storyville. Ce simple synopsis du film du cinéaste français nous porte à réfléchir sur une des réalités du tourisme sexuel. Il nous laisse envisager un fait bien souvent passé sous silence, la prostitution enfantine. L'Amérique a commencé à légiférer contre les abus sur les enfants à partir des années 1875 et une société de protection de la cruauté faite aux enfants commence à s'intéresser aux enfants (garçons et fillettes) travaillant dans les maisons closes dans les années 1890 (Schultz, 1982). Cependant des témoignages oraux recueillis notamment par $\mathrm{Al}$ Rose ont donné matière à Louis Malle pour son film.

De nos jours, Ernest J. Bellocq est considéré comme un exceptionnel témoin de la vie de ces résidentes de maison close. Le livre d'Al rose présente de nombreuses photographies des clichés abîmés pris par ce photographe, vivant au sein de Storyville. Son œuvre, du fait de la nostalgie d'une époque romantisée est très à la mode, tant par les expositions nombreuses qui lui sont consacrées que par les livres et les sites Internet donnant à voir certaines de ces photographies ${ }^{10}$. Il n'est pas inintéressant de noter que son œuvre fascine les plus grands intellectuels américains comme Susan Sontag qui vient de commettre une introduction au dernier livre traitant de Storyville, Bellocq: Photographs from Storyville, the Red-Light District of New Orleans ${ }^{11}$. Ces photos datent d'environ 1912 et montrent de très belles femmes alanguies, fort peu vêtues ou de manière provocante comme marguerite Griffin, attraction principale de la Maison de Mimie White au 221 North Basin. Elles dépeignent une existence orientée vers l'attente du client, dans un décor de soieries et de coussins. Elles nous font prendre conscience de l'environnement différent que pouvait être celui d'une maison close réservée à a bourgeoise et le très épuré garni de la prostituée de la rue.

Il faut bien comprendre que dès la légalisation et officialisation de Storyville, ce quartier devint l'attraction principale du tourisme dans la ville (Asbury, 1940). Il était le lieu de tous les trafics, drogue, alcool 
et jeux. Il était donc une source de revenus économiques énormes qui gravitaient autour de la commercialisation des femmes.

Al Rose a estimé dans son étude que les prostituées, à elles seules, drainaient en 1900 un volume d'affaires de 140000 dollars par semaine ce qui équivaudrait aujourd'hui en dollars constants à plus de 3 millions de dollars ${ }^{12}$. Or, si les plus basses de ces estimations nous paraissent réalistes, compte tenu de tous les commerces illicites ayant pour siège de quartier, on arrive à la somme annuelle de 10 millions de dollars de 1900 soit 22 fois cette somme actuellement.

Il s'avère que tout un monde, régi par l'appât du gain, gravitait autour de la prostituée et de son labeur. Celle-ci était directement exploitée par son souteneur et par la gérante de la maison close, appelée Madam (Wiltz, 2000). Il existait aussi les vendeurs de médecine contre les maladies vénériennes et le policier qui percevait sa redevance. Mais ce quartier rapportait aussi de l'argent aux gens d'affaires les plus établis comme les propriétaires des maisons louées aux Madames, mais également tous les commerçants qui fournissaient directement à domicile et à prix d'or les articles désirés par ces demoiselles.

Quelques figures célèbres ont marqué Storyville par leur qualité de «gestionnaire d'entreprise ». On se doit de citer Josie Arlington, de son vrai nom Mary Deubler, Lulu White, une métisse, et Tom Anderson, le Maire de Storyville. Ce dernier avait su œuvrer à la fois comme tenancier de bars, maisons closes et autres commerces illicites mais aussi comme citoyen impliqué dans la politique locale et reconnu comme une personnalité publique. Hormis ses commerces liés à Storyville, il sut investir dans le pétrole et termina tranquillement sa vie dans une très tolérante Nouvelle-Orléans de 1928.

Les deux femmes avaient ouvert des maisons de luxe. Celle de Lulu White, Mahogany Hall, accueillait des prostituées de sang mêlées dans un décor fastueux dans lequel Jerry Roll Morton s'est produit un temps comme Professeur résident. De nombreuses photos de Bellocq ont été prises dans ce manoir sis au 239 rue Basin. Au 225 de cette même rue se trouvait la demeure de Josie Arlington qui officiait à titre de Madam dans un décor d'une ostentation sans nulle pareille. Toutes ces entreprises usaient de la publicité pour se faire connaître des touristes.

\section{Un temps privilégié, celui du Carnaval et du tourisme sexuel}

Les faiseurs d'images que sont les rédacteurs de guide du XIX ${ }^{\mathrm{e}}$ siècle ont su inspirer les entrepreneurs de Storyville. Ainsi les plus grands renseignements que nous pouvons glaner sur cette époque résident dans les archives constituées par les publicités, que l'historien retrace dans les journaux, et par les quelques fascicules appelés Blue Book, petits guides payants que se procuraient les touristes dès leur arrivée à la Nouvelle-Orléans.

\section{Les Blue Books, guides touristiques spécialisés}

Ce Blue Book, premier guide de Storyville, est daté de 1900. L'intérêt pour ce guide de nos jours est flagrant puisqu'on peut encore actuellement s'en procurer une copie pour cinq dollars en passant par Internet ${ }^{13}$. Cette recherche d'un tel artefact semble dire que les touristes américains éprouvent une forme de nostalgie pour le passé de ce lieu de débauches à saveur française. La NouvelleOrléans, pour ne pas perdre la manne touristique, a su convertir la destination de Storyville en publicisant les attractions de la rue Bourbon. Il s'agit de comprendre que l'imaginaire se nourrit de toutes sortes de documents et que Storyville fait partie de la mémoire collective américaine comme une espace de licences et de liberté. La rue Bourbon est une pâle réplique de ce qu'était Storyville, toutefois cette rue est un construit culturel attrayant pour des clients américains parce qu'il est exacerbé par les souvenirs et la réputation du lieu originel fréquenté par d'autres américains précurseurs.

Ces Blue Books, à ne pas confondre avec les guides de cotations automobiles contemporains, présentaient, sur une quarantaine de pages, des annonces publicitaires vantant les lieux de plaisirs sans jamais faire une allusion directe à des services sexuels. L'initiative de la publication de ce guide revient au Maire de Storyville, Tom Anderson.
Il utilisait un certain Billy Struve comme secrétaire de rédaction, un ancien journaliste du New Orleans Item. Celui-ci rédigea tous les fascicules et il est intéressant de se pencher sur sa prose, particulièrement sur les introductions qu'il fit pour les six éditions successives. La première est un chef d'œuvre de déculpabilisation du lecteur masculin. Il écrit en substance : «À quoi sert de vivre si vous ne profitez pas de la vie » et indique que la mort sera là ensuite pour toujours. Il donne ensuite la recette pour savoir profiter de la vie : lire attentivement le Blue Book. Dans cette première édition, un certain nombre d'expressions argotiques et typiques font leur apparition, comme la qualification des établissements par le terme de Sporting Houses, formulation qui se prêtait aux maisons de jeux et qui là prenait une connotation plus large incluant, dans la part de risque, les maisons de prostitutions. Le quartier est surnommé le Tenderloin, tendre filet, euphémisme pour indiquer le quartier du vice. Ce Tenderloin se voit adjoindre le nombre 400, supposément le nombre d'établissement dans ce quartier contrôlé par Anderson. Il donne une indication intéressante sur période pré-Storyville puisqu'il signale qu'il existait un vieux Anderson County, espace de prostitution contrôlé par le jeune anderson, situé au cœur même du French Quarter, délimité entre les rues Rampart, Dauphine, StPierre et Customhouse (Figure 1).

Dans ces différents guides, l'auteur utilise des méthodes de repérage simples mais efficaces. Comme on le fait pour les guides voulant souligner un lieu à visiter en priorité, le rédacteur utilise un système d'étoiles pour indiquer les maisons où l'on consomme exclusivement du vin en compagnie des plus belles femmes. Au fil des éditions on voit qu'il met en place un système de lettres pour indiquer la race ou l'origine des filles présentées dans ces établissements. On peut retrouver des listes où l'on voit des $\mathrm{W}$ pour Blanches, $\mathrm{C}$ pour femmes de couleur, O pour Octoroon ou Métisse, $\mathrm{J}$ pour Juive et $\mathrm{F}$ pour Française. L'édition de 1906 est fort intéressante car le rédacteur émet une justification de l'existence et du guide et de Storyville. Celui-ci est présenté comme un quartier distinctif : en comparaison des autres Red Lights américains, il est le seul couvert par la Loi. Par sa régulation interne le district protège le touriste des mauvais coups. Le 
guide est informatif et donne un gage de qualité aux établissements y souscrivant une publicité. Peu à peu, un grand nombre de photos sont inclues dans le fascicule qui devient un vrai annuaire de cent pages dans lequel les dancings et cabarets des années 1910 rejoignent les publicités des maisons closes mais aussi les prêteurs sur gage, les vendeurs de produits de cure pour lutter contre les maladies vénériennes ou les entrepreneurs de pompes funèbres.

Les principales annonces restent celles des Maisons de Tolérance. Chaque établissement est présenté selon sa spécialité et la réputation de la Madam qui donne un style à son établissement et aux filles qu'elle recrute. On remarque des permanences puisque les maisons sont toujours meublées avec splendeur et une certaine ostentation, les faisant ressembler à des musées ou des scènes d'exposition, telles des décors rococos de foires internationales. Les femmes sont réputées pour leur beauté et leur entrain, ce sont les Jolly good fellows, des « ladies stylées qui sont recherchées par les hommes de New York à la Californie ». Elles sont capables de redonner la joie de vivre aux messieurs seuls, touristes d'un temps limité dans la ville. Les Comtesses et autres altesses de la nuit vivent dans des manoirs ou des châteaux et ouvrent leurs lieux à toute personne digne de s'y présenter. La Reine Parisienne de l'Amérique, Emma Johnson, dans sa maison de toutes les Nations, rivalise avec la Comtesse Willie Piazza et sa maison de joie. Cette dernière, une métisse octoroon, était reconnue comme une des femmes les plus cultivées du Vieux Carré. Elle était la reine de la mode à défaut d'être une véritable comtesse. Elle aimait la musique et faisait jouer dans sa demeure des artistes tels que Jerry Roll Morton ou Tony Jackson. Emma Johnson était connue pour ses spectacles de cirque, véritables tableaux vivants sexuels.

\section{Les publicités légales et l'exploitation du phénomène durant la période carnavalesque.}

Le grand moment touristique de la NouvelleOrléans, aujourd'hui comme il y a un siècle, est la période de Carnaval avec le clou de son défilé à Mardi Gras. Cette tradition ca- tholique reprise à des fins commerciales est extrêmement connue de nos jours. Les Américains, une fois devenus les maîtres de la Louisiane et surtout après la guerre de Sécession, ont repris à leur compte cette tradition ainsi qu'un certain nombre de légendes qui entouraient les mœurs françaises et créoles de la période esclavagiste. Il n'est pas inutile de rappeler que ce sont des étrangers à la ville qui ont concouru à la réputation de la Nouvelle-Orléans comme un lieu de luxure où les Maîtres blancs couchaient avec leurs esclaves de couleur. Dans cette NouvelleOrléans du XVIII ${ }^{\mathrm{e}}$ siècles, les Français ou Canadiens-français se choisissaient des maîtresses attitrées parmi les plus belles métisses durant le Bal des Octoroons, jeunes femmes possédant un huitième de sang noir. Cette tradition était appelée le plaçage. Celles-ci leur donnaient des enfants qui bien souvent étaient affranchis ainsi que leur maîtresse. Ces femmes vivaient en concubinage notoire et leurs maîtres les installaient dans de luxueuses demeures dans le Faubourg Marigny, quartier voisin du Vieux Carré, séparé par l'avenue de l'Esplanade.

Ces mœurs différentes, jugées scandaleuses par la bonne société protestante a toutefois contribué à exalter un interdit qui a nourri un goût de la transgression chez les touristes des autres États lorsqu'ils sont venus découvrir ces lieux auréolés de tant de permissivité. Le carnaval rendait la transgression possible. $\mathrm{Ce}$ temps d'inversion des comportements ordinaires se fondait dans un lieu exotique, loin du monde de l'habitude protestante aux mœurs puritaines. Ici les parades dans les rues, les bals masqués soutenus par des organisations secrètes ont su auréoler le lieu d'un parfum désirable.

Le plus célèbre moment était le jour de Mardi Gras, lors du bal masqué « Ball of the Two Well Known Gentlemen » institué à partir de 1882. Ce bal est bien documenté, car il fut extrêmement publicisé, tout particulièrement dans le journal à scandales The Mascot. Ce bal privé, organisé par un club du même nom, réunissait des proxénètes, des demi-mondaines, des politiciens véreux, des trafiquants et des policiers corrompus. D'autres bals concurrents furent mis sur pied par d'autres clubs privés aussi mafieux comme le C.C.C. Club ou le Red
Light Social Club dirigé par le chef de la police David Hennessey. Malgré l'assassinat de ce dernier, ces bals redoublèrent. On les voit sous l'appellation de French Balls. Le journal Mascot publiait des reportages persifleurs sur les liens entre le milieu des affaires et les trafiquants locaux sous le regard bienveillant d'une police corrompue. Ainsi dans le Sunday Sun de 1906 on pouvait découvrir le nom des postulantes, Madam notoires comme Josie Arlington, Margaret Miller ou Flora Meeker et bien d'autres.

Al Rose prétend que ce type de bal était plus couru que les attractions plus conventionnelles entourant les parades des Krewes of Rex, Comus et Momus (Rose: 64) et que les invitations gratuites pour les femmes étaient fort difficiles à se procurer car même les jeunes femmes de la bonne société intriguées par cette mode cherchaient à y participer profitant des masques pour ne pas se faire reconnaître. Il ne faudrait pourtant pas croire que tout les résidents de la Nouvelle-Orléans appréciaient ce quartier. Loin s'en fallait. Mais, tout comme aujourd'hui dans le vieux Carré où les habitants se plaignent des nuisances que procurent la rue Bourbon et les hordes de touristes se massant pour le Carnaval autour de la Cathédrale St-Louis, les résidants de la Nouvelle-Orléans, d'il y a un siècle, souffraient ce quartier légalisé qui rapportait un essor économique à la ville.

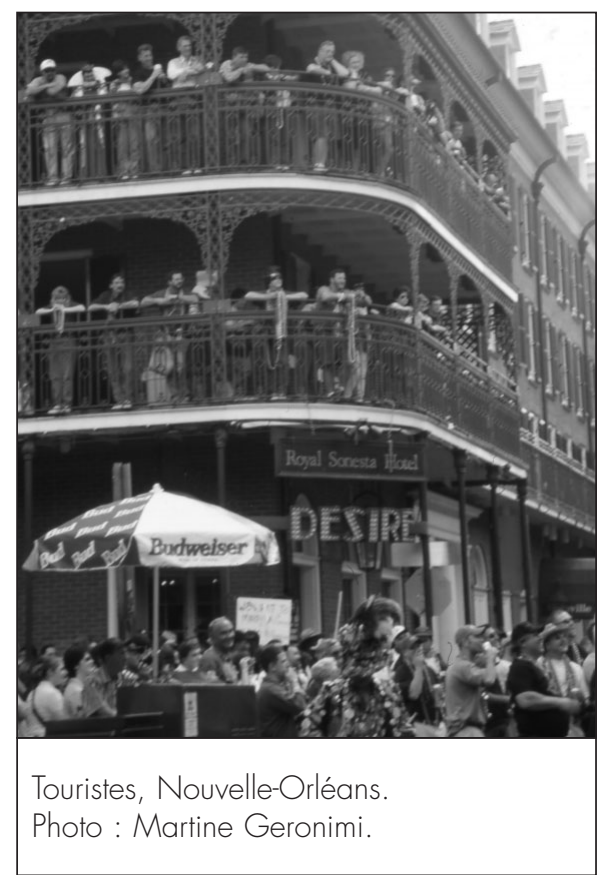




\section{Conclusion}

Les Red Lights comme le tourisme sexuel sont des sujets importants et d'actualité dans notre société de 2003. La Nouvelle-Orléans, il y a un siècle, à titre d'espace festif précurseur, a mené une expérience d'encadrement de la prostitution en légalisant une portion de sa ville et en la dotant d'un statut d'avant garde. Prônée par un mouvement réformiste, qui voulait réglementer le phénomène grandissant de la prostitution et en même temps préserver la population de la multiplication du vice sous les yeux des honnêtes citoyens, l'expérience a eu de nombreuses conséquences que nous ne pouvons ignorer.

L'une, et non la moindre, est la légitimation d'une activité qui existait de façon souterraine. L'autre semble la limitation des crimes crapuleux, puisque les activités auparavant illicites étaient limitées à une portion restreinte de la ville et sous contrôle des « gens d'affaires » et des policiers bienveillants. Bien entendu, ces constations objectives ne doivent pas masquer des réalités plus négatives.

La première est une double ghettoïsation de l'espace de la ville, à la fois espace réservé et approprié dans un but de spécialisation des activités mais également ghettoïsation sociale. En effet, en ce temps de ségrégation raciale, les maisons de tolérance étaient légalement scindées selon l'appartenance ethnique. Non seulement les femmes blanches, de couleur ou métisses ne pouvaient cohabiter mais une maison de femmes noires ne pouvaient être sous le patronage d'un homme de couleur. De plus, en février 1917, peu avant la décision de fermeture du quartier par le Fédéral, une ordonnance de la mairie renforça la ségrégation en limitant Storyville aux Maisons closes réservées uniquement aux Blancs, clients et prostituées. Les gens de couleur étaient forcés à s'installer dans un quartier de l'autre côté de Canal (Uptown) ce qui aurait contraint des Madams Octoroons comme Lulu White ou Willie Piazza à se relocaliser si le fédéral n'était intervenu définitivement.

La seconde est le développement d'une véritable industrie du tourisme sexuel et non pas la régression de la prostitution mais une forme de canalisation des retombées du commerce du sexe et une industrialisation des méthodes. Ainsi la publicisation intense au travers de toute l'Amérique d'une vision paradisiaque de la Nouvelle-Orléans avec ses belles Créoles et ses patios comme dans le Newly Harper se conjoignait avec les vertus du bouche à oreille et des Blue Books acquis sous le manteau ou ramenés par des visiteurs précurseurs. Il est très difficile de trouver des données sur le nombre de visiteurs fréquentant effectivement Storyville, mais on a pu comprendre que la prostitution et les commerces illicites rapportaient des sommes considérables alimentées par les visiteurs étrangers.

Après la fermeture de Storyville, malgré l'interdiction de la prostitution, le commerce du sexe repris de plus belle surtout dès le retour des soldats qui reprirent leur place dans le monde du travail. Aussi robert Tallant pouvait dire de la NouvelleOrléans qu'elle pouvait se comparer au pire des quartiers de Marseille, Honolulu ou Singapore (Tallant, 1950). Dans les années 1920 et 1930 , le commerce du sexe demeura l'une des principales sources de revenus pendant la Grande Dépression. Le Blue Book fut enfin remplacé par les guides écrits par Robert Kinney, intitulés The Bachelor in New Orleans (Kinney, 1942).

\section{Martine Geronimi est détentrice d'un} PhD en sciences géographiques de l'Université Laval. Elle enseigne la géographie touristique et la géographie historique et culturelle à l'UQAM. Sa thèse a porté sur les paysages patrimoniaux et touristiques des villes de mémoire française : le Vieux-Québec et le Vieux Carré à la Nouvelle-Orléans.

\section{Notes}

1 Les mœurs dissolues des Créoles de la Nouvelle-Orléans ont été largement publicisés auprès du public américain par l'auteur George Washington Cable. De même les guides touristiques colportaient les relations charnelles des maîtres blancs d'origine française avec leurs esclaves de couleur.

2 Notamment quelques guides touristiques appelés Blue Book

3 Après la fin de la guerre de Sécession, la Nouvelle Orléans retrouve une forte expansion économique stimulée par les
Grandes Expositions comme celle du World's Industrial Cotton Centenial de 1884 sur le site duquel sera construit le Parc Audubon.

4 Le plus grand Marché d'esclaves des États Unis (100 000 esclaves vendus), qui s'est tenu jusqu'en 1862, se déroulait à l'intérieur des hôtels comme l'Hotel St-Louis dans le Vieux Carré ou l'hôtel St-Charles, côté américain. Lire Walter Johnson, Soul by Soul: Life Inside the Antebellum Slave Market. Cambridge, Mass.: Harvard University Press, 1999.

5 La prostitution est attestée se diffuser du vieux Carré vers les quartiers américains au delà de la rue Canal. De même près du port, non loin de la communauté des Ursulines, la rue Gallatin comportait de nombreux bouges. Cette rue fut elle aussi éradiquée de la mémoire des NéoOrléanais.

6 Je fais ici référence aux thèses d'Eric Hobsbawm concernant l'invention de nos traditions présentes à la fin du $\mathrm{XIX}^{\mathrm{e}}$ siècle.

7 traduit par nos soins

8 à lire sur Internet « thousands of fatherless girls are compelled to choose between a life of shame and starvation» Declaration of Rights for WomenIndependence Hall, Philadelphia, July 4, 1876 http://www. huntington.org/vfw/orgpub/declarations/de cphiladelphia.html

9 texte original sur Internet : www.admin. $\mathrm{ch} / \mathrm{ch} / \mathrm{f} / \mathrm{rs} / \mathrm{i} 3 / 0.311 .31$.fr.pdf

10 Consulter : http://www.corpse.org/issue_ 10/gallery/bellocq/;http://www.dazerea der.com/bellocq.htm

11 http://www.masters-of-photography. com/B/bellocq/bellocq_articles2.html ;

12 Aujourd'hui 5 \$ de 1900, le prix des services d'une prostituée dans une maison close, équivaudrait à 113 \$ d'après ta table de conversion du Columbia Journalism Review.

13 chez : Buddy Stall 4508 St. Mary Street Metairie, LA 70006 OU EN PASSANT PAR https://fbpa.baileylink.net/bldc/bud dystall-com/order.html

\section{Bibliographie}

Asbury, Herbert (1936), The French Quarter. An Informal History of The New Orleans Underworld. Garden City, New York, Garden City Publishing Co. Inc

Boyer, M-C. (1994), The City of Collective Memory: Its Historical Imagery and Architectural Entertainments, Cambridge, MIT Press. 
Bryan, Violet Harrington (1993), The myth of New Orleans in literature: dialogues of race and gender Knoxville, University of Tennessee Press.

Cable, G.W. (1886), The Creoles of Louisiana, New York, C. Scribner's sons.

Cable, M. (1984), Lost New Orleans, New York, American Legacy Press.

Castellanos, Henry C. (1895), New Orleans as it was, New Orleans, L.Graham et Son Ltd.

Chase, J.C. (1949) Frenchman, Desire, Good Children, and Other Streets of New Orleans, New Orleans, Robert L. Crager \& Company.

Christian, Marcus, Archives, University of New Orleans, série XIII, Box 13.

Coleman, W. H. (1885), Historical Sketch Book and Guide to New Orleans and Environs with Map, edited and compiles by several leading writers of the New Orleans Press, New York Will H. Coleman.

Curtis, N. C. (1933), New Orleans : Its Old Houses, Shops and Public Buildings, Philadelphia, J.B. Lippincott.

Curtis, Valdo J. (1879), Illustrated Visitor's Guide to New Orleans, New orleans, J. Curtis Ed.

Federal Writers's Project of the Louisiana of the WPA (1938), Ed. New Orleans City Guide. Cambridge, Riverside Press.

Garvey, Joan B. et Widmer, Mary L. (1982), Beautiful Crescent. A history of New Orleans. New Orleans, Garmer press Inc.

Geronimi, M. (2002), « Le French Market à la Nouvelle Orléans ; alibi patrimonial et mise en scène d'un espace préservé », Ethnologies, 24-1.

Geronimi, M. (2001a), Imaginaires français en Amérique du Nord. Géographie comparative des paysages patrimoniaux et touristiques $d u$ Vieux-Québec et du Vieux Carréà La NouvelleOrléans, Sainte-Foy, Université Laval, département de géographie, thèse de doctorat.

Geronimi, M. (2001b), « Parade zouloue et carnaval indien un mardi gras différent à la Nouvelle-Orléans », Ethnologie.

Geronimi, M. (2001c), « Imaginaire français en Amérique du Nord : Genèse d'un tourisme de distinction à Québec et la Nouvelle-Orléans », Anthropologie et Sociétés 25-2.

Halbwachs, Maurice (1968), La Mémoire collective, Paris, Presses universitaires de France.

Hearn, L. (1873), An American Miscellany, New York, Dodd Mead.Howells.

Hirsch, Arnold R. et Logdson, Joseph (1992), Creole New Orleans. Race and Americanization, Baton Rouge et London, Louisana State Press University, p. 45.
Hobshawm, E. et Ranger, T. (1983), The Invention of Tradition, Cambridge, London N.Y., Cambridge University Press.

Jackson, J. J. (1993), New Orleans in the Gilded Age. Politics and Urban Progress, New Orleans, Louisiana Historical Association.

Johnson, Walter (1999), Soul by Soul: Life inside the Antebellum Slave Market, Cambridge, Mass.: Harvard University Press.

Kennedy, Richard S. (ed.) (1992), Literary New Orleans : essays and meditations, Baton Rouge, Louisiana State University Press.

Kinney, Robert (1942), The bachelor in New Orleans : a handbook for unattached gentlemen and ladies of spirit visiting or resident in the Paris of America, New Orleans : Bob Riley Studios.

Lewis, Pierce F. (1976), New Orleans: The making of an urban landscape, Cambridge, MA, Ballinger ed.

Lillard, John B. (1884), Visitor's Guide to the World's industrial and Cotton Centennial, Louisville, Courrier-Journal Job Printing Co.

McGinn, Th. A. J. (1998), Prostitution, Sexuality, and the Law in Ancient Rome, Oxford.

MacFarlane, W.G. (1928), The Charms of the Vieux Carré, the Mardi Gras, the Color and Romance of French and Spanish Days, Chicago, American Autochrome.

Mildo Hall, G. (1995), Africans in Colonial Louisiana, Baton Rouge et London, Louisiana State University Press, p. 10.

Moynihan, Ruth Barnes (1993), Second to None : A Documentary History of American Women : From 1865 to the Present, Vol 2, Lincoln, NB: University of Nebraska Press.

Rose, A. (1974), Storyville, New Orleans. Being an authentic Illustrated Account of the Notorious Red-Light District, Tuscaloosa and London, University of Alabama Press.

Rosen, Ruth (1990), Lost Sisterhood: Prostitution in America, 1900-1918, Johns Hopkins University Press.

Schultz, L. G. (1982), « Child sexual abuse in historical perspective ", Journal of Human Sexuality and Social Work, 1(1), 21-35.

Selwyn, T. (1996), The Tourist Image: Myth and Myth Making in Tourism, New York, John Wiley and Sons.

Stebbins, R. (1995), The Connoisseur's New Orleans, Calgary, University of Calgary Press.

Tallant, Robert (1950), The Romantic New Orleanians, New York, Dutton.

The Picayunes's Guide to New Orleans (1896), Tourist's Guide-Book to the City of New Orleans, New Orleans, La., Nicholson \& Co.

Tregle, J. (1992), «Creoles and Americans », dans Arnold R. Hirsch et Joseph Logsdon (eds), Creole New Orleans: Race and Americanization, Baton Rouge, Louisiana State University Press, pp. 131-185.

Van Gennep (1909), Les rites de passage, Paris, Librairie Critique Émile Nourry.

Vanoyeke, V. (1990), La prostitution en Grèce et à Rome, Paris.

Veblen, Thorstein (1899), The Theory of the Leisure Class, Macmillan (édition française Gallimard -1969 -, Théorie de la classe de loisir, traduit par Louis Évrard, précédé de « Avezvous lu Veblen? », par Raymond Aron).

Vesey, C. et Dimanche, F. (2003), From Storyville to Bourbon Street: Vice, Nostalgia, and Tourism, Université de la Nouvelle-Orléans (à paraître).

Waldo, J. Curtis (1875), Visitor's Guide to New Orleans, November 1875, New Orleans, J Curtis Waldo Southern Publishing and Advertising House.

Walkowitz, Judith (1980), Prostitution and Victorian Society, Cambridge, University Press (cité par Megara Belle dans the Fallen Woman in Fiction and Legislation).

Wilson, S. Jr. (1968b), Vieux Carré Historic District Demonstration Study, New Orleans, City of New Orleans.

Wiltz, Christine (2000), The Last Madam: A Life in the New Orleans Underworld, New York, Faber \& Faber.

Winnifrith, Tom (1994), Fallen Women in the Nineteenth-Century Novel. St. Martin's Press.

Zukin, S. (1995), The Cultures of Cities, Oxford Cambridge, Blackwell Publisher. 